\title{
In Silico Screening of Circulating MicroRNAs as Potential Biomarkers for the Diagnosis of Ovarian Cancer
}

\author{
Lei Wu, ${ }^{1,2}$ Wenwen Shang, ${ }^{1,2}$ Hong Zhao, ${ }^{1,2}$ Guodong Rong, ${ }^{1,2}$ Yan Zhang, ${ }^{1,2}$ Ting Xu, ${ }^{1,2}$ \\ Jiexin Zhang, ${ }^{1,2}$ Peijun Huang, ${ }^{1,2}$ and Fang Wang $\mathbb{D}^{1,2}$ \\ ${ }^{1}$ Department of Laboratory Medicine, The First Affiliated Hospital of Nanjing Medical University, 210029 Nanjing, China \\ ${ }^{2}$ National Key Clinical Department of Laboratory Medicine, 210029 Nanjing, China \\ Correspondence should be addressed to Fang Wang; wangfang@njmu.edu.cn
}

Received 20 March 2019; Revised 2 July 2019; Accepted 16 July 2019; Published 4 August 2019

Academic Editor: Maria Dalamaga

Copyright @ 2019 Lei Wu et al. This is an open access article distributed under the Creative Commons Attribution License, which permits unrestricted use, distribution, and reproduction in any medium, provided the original work is properly cited.

\begin{abstract}
Current screening tests for the diagnosis of ovarian cancer (OC) face enduring challenges. However, microRNAs (miRNAs) are stable in the circulation and may be promising molecular biomarkers for OC prediction. Circulating miRNA expression profiles in OC were analyzed using sequencing data from the Gene Expression Omnibus database. Differentially expressed miRNAs were generated from GSE94533, of which some were selected as candidate miRNAs based on an electronic search of the literature and comprehensive evaluation. A meta-analysis was preformed to integrate an evaluation index for these miRNAs in diagnosing OC patients. An independent validation set (GSE106817) was also conducted to further confirm the roles of these miRNAs. We identified four MIR200 members (MIR200A, MIR200B, MIR200C, and MIR429) and MIR25 as being differentially expressed among malignant or benign ovarian tumor patients and healthy controls. In the meta-analysis, these five miRNAs yielded a pooled area under the receiver operating characteristic (ROC) curve (AUC) of 0.78 (sensitivity: 64\%, specificity: 88\%) in discriminating OC from healthy controls, while the four MIR200 members demonstrated a summary AUC of 0.81 (sensitivity: 92\%, specificity: 69\%) in differing OC cases from patients with benign disease. In the validation set, differential expression and ROC curve analyses of these miRNAs were consistent except for MIR25. The circulating MIR200 family has the potential to become reliable and noninvasive biomarkers for OC diagnosis. Studies with larger cohorts are warranted to validate the applicability of these miRNAs.
\end{abstract}

\section{Introduction}

Ovarian cancer (OC) is one of the most common and lethal gynecological malignancies and is the fifth leading cause of cancer death in females worldwide. Indeed, OC was reported to account for $2.6 \%$ and $5.0 \%$ of the total cancer incidence and deaths in women, respectively [1]. Most OC patients are diagnosed at late stages, and the 5-year survival rate is $46.5 \%$ based on the Surveillance, Epidemiology, and End Results Program, highlighting the need for an effective screening strategy. CA125 is the current serum biomarker for detecting and monitoring $\mathrm{OC}$ in clinical practice, but this is usually only elevated in advanced disease and has poorer sensitivity for early-stage cancer. Additionally, CA125 has limited specificity, and elevated levels do not always reflect a diagnosis of OC but can be indicative of endometriosis, liver cirrhosis, pelvic inflammatory disease, and some benign neoplasms [2,3]. Thus, there is an urgent need to explore sensitive, noninvasive biomarkers for the detection of OC.

Accumulating evidence has shown that microRNAs (miRNAs) function as oncogenes or tumor suppressor genes. miRNAs are short (18-24 nucleotide) noncoding RNAs that regulate gene expression at the posttranscriptional level through base pairing with complementary sequences of the $3^{\prime}$-untranslated region of mRNA. Expression of miRNAs is aberrant in various types of cancers where they are readily detected in circulating body fluids such as serum or plasma [4]. Taylor and Gercel-Taylor previously isolated eight exosomal miRNAs (MIR21, MIR141, MIR200A, MIR200B, MIR200C, MIR203, MIR205, and MIR214) in the blood of OC patients that were upregulated in comparison with benign controls, which may reflect tumor profiles and aid 
diagnosis [5]. Following this investigation, a series of profiling studies were carried out to explore the diagnostic potential of extracellular miRNAs. Kan et al. identified a small marker panel combining MIR200B and MIR200C as a positive classifier of OC [6], while Langhe et al. showed that four miRNAs (MIRLET7I, MIR122, MIR152, and MIR25) were significantly downregulated in OC patients and could discriminate benign from malignant ovarian disease [7]. More recently, Elias et al. proposed a pattern of seven microRNAs (MIR29A, MIR92A, MIR200C, MIR320C, MIR335, MIR450B, and MIR1307) in the blood that appears to predict OC [8].

In this study, we profiled the global expression patterns of serum miRNAs in OC utilizing next-generation sequencing and clinical data from the Gene Expression Omnibus database (dataset ID: GSE94533). Considering the inconsistencies across different studies of the role of miRNAs in OC, we also performed systematic analysis combined with a validation study (GSE106817) to evaluate the diagnostic efficiency of circulating miRNAs with the aim of identifying a novel class of noninvasive biomarkers for the diagnosis of OC.

\section{Materials and Methods}

2.1. Next-Generation Sequencing Data and Differential Expression Analysis. miRNA profiling data were obtained from GSE94533 [8]. The corresponding platform was the Illumina NextSeq 500 system, and sequence tags were mapped to miRBase 20. Expression levels were quantified in transcripts per million. Using raw count data, the edge $\mathrm{R}$ package of $\mathrm{R}$ was utilized to identify differentially expressed miRNAs (DEmiRNAs). A false positive rate $<0.05$ and $\mid \log _{2} \mathrm{FCl}$ (fold change) $>1$ were set as cutoff points to screen out DEmiRNAs.

2.2. Systematic Review and Meta-Analysis. We conducted a literature search for all studies that evaluated the diagnostic value of candidate circulating miRNAs for $\mathrm{OC}$ in the PubMed database up to January 2019. The following key terms were used in the search: "circulating" or "serum" or "plasma", "DEmiRNAs screened above", "Ovarian" and "cancer" or "carcinoma" or "neoplasm". References cited in each retrieved article were also manually scanned to identify additional eligible studies.

Articles were recruited for this meta-analysis if they fitted the following criteria: (1) investigated the diagnostic value of circulating miRNAs in OC, (2) peripheral blood for miRNA analysis was collected before any treatment, (3) sufficient data to construct a $2 \times 2$ contingency table, and (4) published in English. The following data characteristics were collected for each included article: first author's name, publication year, country of publication, sample type, detecting method, sample size, and data for the two $2 \times 2$ contingency tables (sensitivity, specificity, area under the ROC curve (AUC), and corresponding $95 \%$ confidence interval (95\% CI)).

2.3. Validation of Candidate miRNA Expression. The expression levels of candidate miRNAs were subsequently validated in an independent cohort (GSE106817) [9]. In this set, a total of 3938 serum samples were analyzed by miRNA microarray, including 320 OC, 2759 noncancer controls, and 859 other solid cancers. Comprehensive miRNA expression analysis was evaluated using the $3 \mathrm{D}-\mathrm{Gen} \mathrm{e}^{\circledR}$ Human miRNA Oligo Chip (Toray Industries Inc., Tokyo, Japan). Each miRNA signal value was standardized using the ratio of the average signal value of the three internal control miRNAs (miR-149-3p, miR-2861, and miR-4463) to the preset value.

2.4. Functional Annotation and Enrichment Analysis for Candidate miRNAs. The potential targets of candidate DEmiRNAs were obtained using the miRTarBase database (http://mirtarbase.mbc.nctu.edu.tw/). We only included target genes that were validated by at least two of the following experimental methods: reporter assay, western blot, quantitative PCR, microarray, and next-generation sequencing experiments. Gene ontology (GO) enrichment and Kyoto Encyclopedia of Genes and Genomes (KEGG) pathway analysis were performed by the Database for Annotation, Visualization and Integrated Discovery (DAVID; https:// david.ncifcrf.gov/) online tool. A Benjamini $P$ value of $<0.05$ was used to indicate a statistically significant difference in the above pathway enrichment analysis.

2.5. Statistical Analysis. Data analysis was performed using Stata 13 (Stata Corporation, College Station, TX) and R (version 3.1.3) software. MIDAS modules for Stata were used to estimate the pooled specificity, sensitivity, and AUC of the summary receiver operating characteristic (SROC) [10]. Heterogeneity among studies was estimated with the $Q$ test and $I^{2}$ statistics, and $I^{2}>50 \%$ indicated the existence of significant heterogeneity. The presence of publication bias was detected using the Deeks funnel plot asymmetry test. Different distributions of relative serum miRNA expression levels between OC cases and controls in GSE94533 and GSE106817 were compared using the Mann-Whitney $U$ test. ROC curve analysis was performed to calculate the AUCs to evaluate the associations of candidate miRNAs and OC. Tests of significance were two-tailed, and a $P$ value $<0.05$ was considered statistically significant.

\section{Results}

3.1. Differentially Expressed Serum miRNAs. GSE94533 included 98 OC patients, 21 patients with borderline tumors, 45 patients with benign ovarian diseases, and 15 healthy women. Of the OC patients, $44.9 \%$ presented at stage III or IV disease, as defined by the International Federation of Gynecology and Obstetrics (FIGO). We used a five-set Venn diagram to demonstrate the common differentially expressed miRNAs (Figure 1(a)). A total of 81 DEmiRNAs were identified between stage I/II cancer and healthy controls, including 50 upregulated and 31 downregulated; 240 DEmiRNAs were identified between stage III/IV cancer and healthy controls, including 172 upregulated and 68 downregulated; 77 DEmiRNAs were identified between borderline tumors and healthy controls, including 52 upregulated and 25 downregulated; 115 DEmiRNAs were identified between invasive cancers and benign lesions, including 87 upregulated and 28 downregulated; and 74 DEmiRNAs were identified 


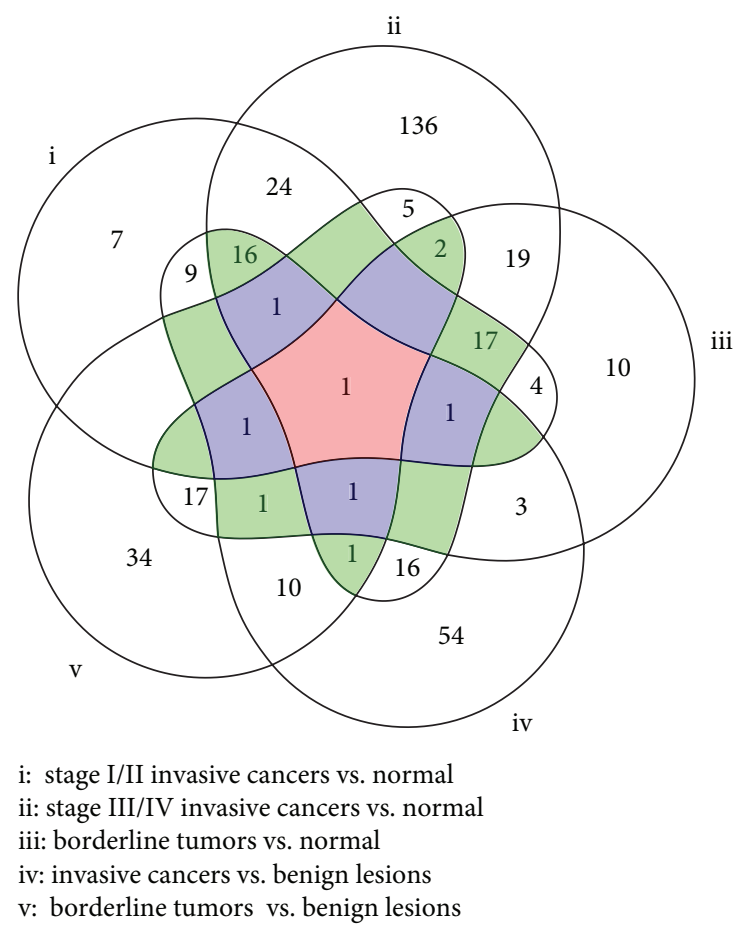

(a)

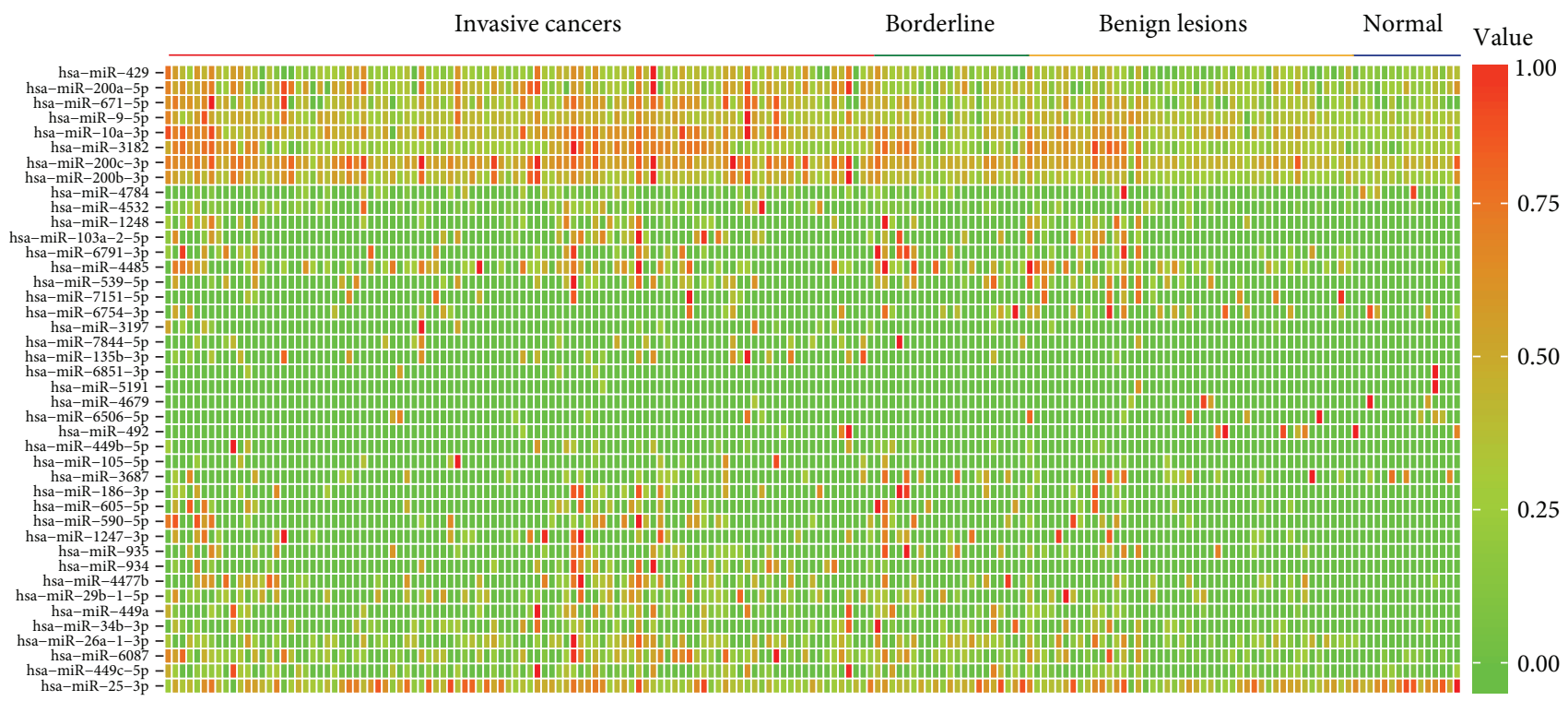

(b)

FIGURE 1: Identification of differentially expressed miRNAs in serum. (a) Five-set Venn diagram showing common differentially expressed miRNAs. (b) Heat map illustrating common miRNA profiles.

between borderline tumors and benign lesions, including 43 upregulated and 31 downregulated miRNAs. A total of 42 DEmiRNAs were common to three or more sets. Of these, 32 were upregulated and 10 were downregulated in invasive cancers or borderline tumors compared with benign lesions and healthy controls (Figure 1(b)).

3.2. Determination of Candidate miRNAs and Diagnostic Accuracy in OC. To further evaluate the clinical applicability of the 42 common DEmiRNAs for the diagnosis of OC, we screened all available studies based on the above search strategy and identified five miRNAs (MIR200A, MIR200B, MIR200C, MIR429, and MIR25) as being reported so far. We consequently focused our attention on their expression in GSE94533. As shown in Figure 2(a), miR-200b-3p, miR200c-3p, and miR-429 serum expression was significantly higher in OC than in healthy controls, while the serum expression of miR-25-3p was extremely low in OC patients. 


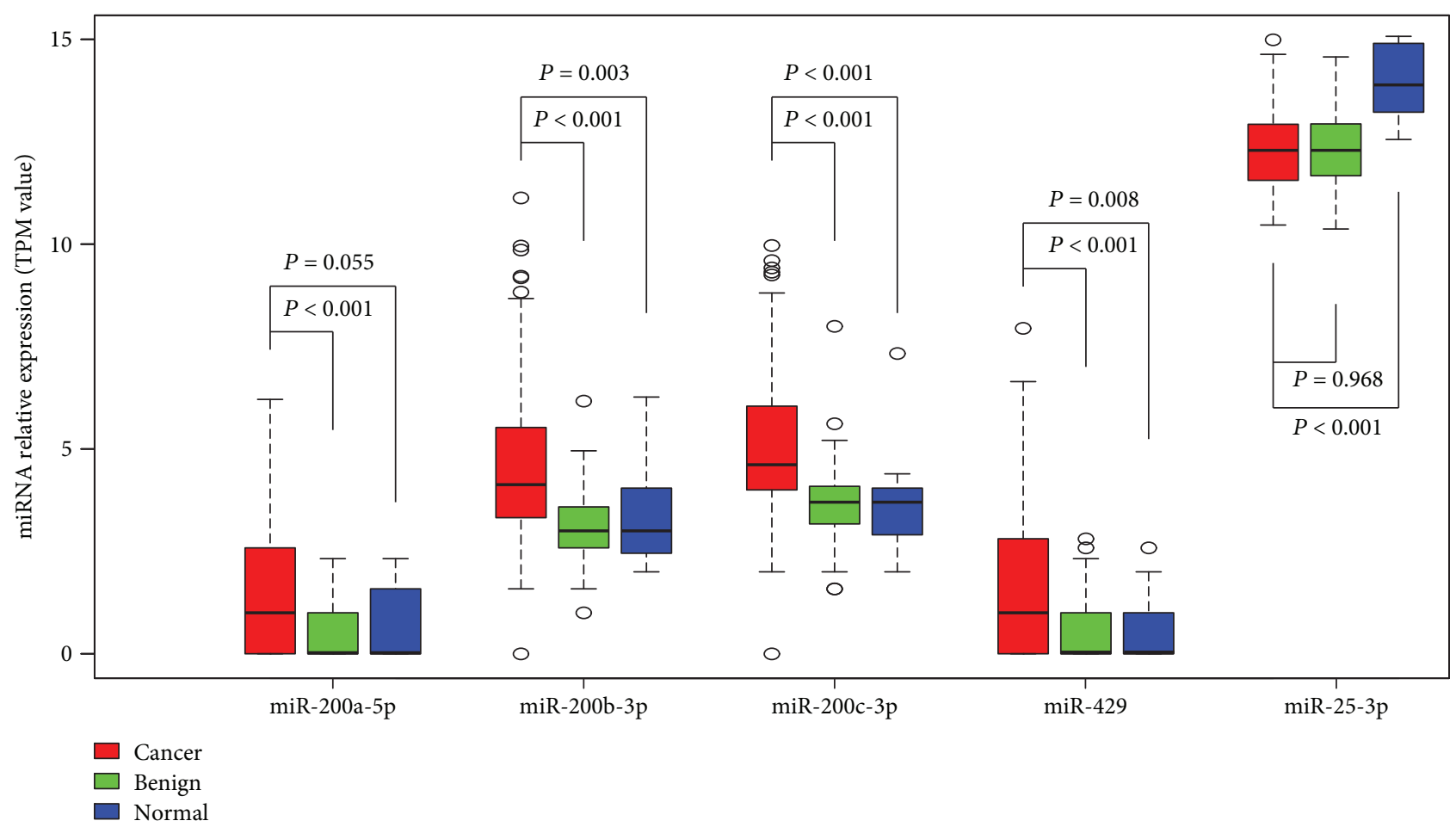

(a)

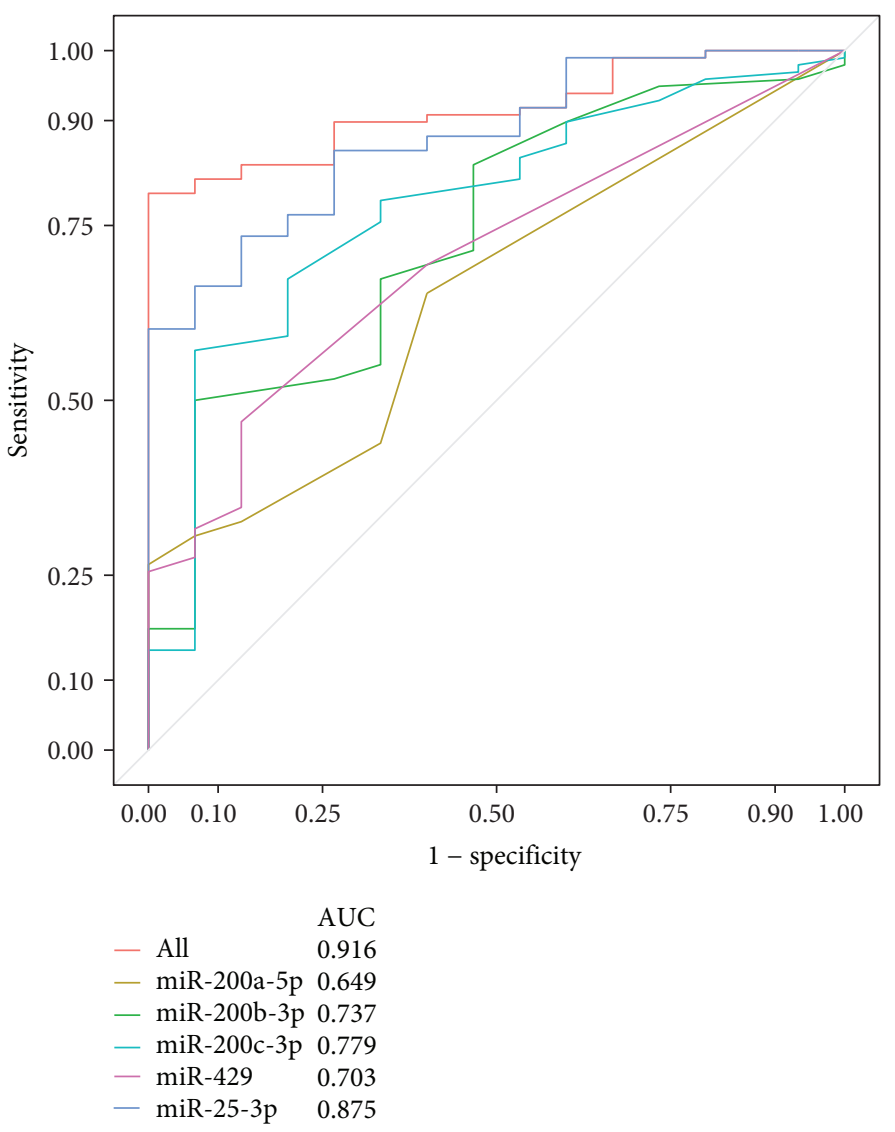

(b)

FIgURe 2: Continued. 


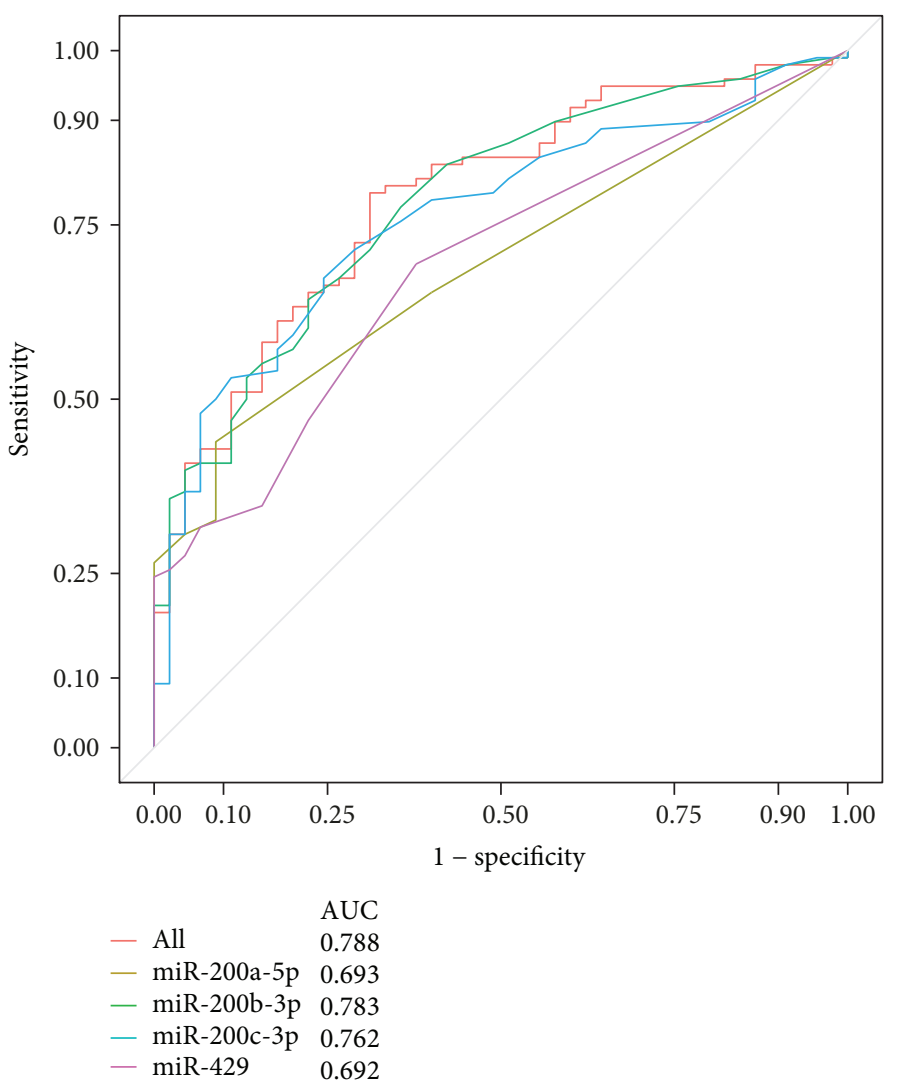

(c)

FIGURE 2: Quantification of candidate miRNAs in the serum of healthy women, patients with benign ovarian diseases, and those with ovarian cancer. (a) Box plot comparing miRNA concentrations in the serum of healthy women, patients with benign ovarian diseases, and those with ovarian cancer. (b) Receiver operating characteristic curve for candidate miRNAs showing its potential to discriminate ovarian cancer patients from healthy women. (c) Receiver operating characteristic curve showing the profiles of sensitivity and specificity of candidate miRNAs to distinguish ovarian cancer from benign ovarian diseases.

Additionally, the expression of miR-200a-5p, miR-200b-3p, miR-200c-3p, and miR-429 was higher in OC patients compared with those with benign lesions.

To reflect the diagnostic potential of the selected miRNAs, ROC curve analysis was performed. We used information on the cutoff from each study to determine an optimal cutoff by maximizing the Youden index (sensitivity + specificity -1$)$. The relative expression of serum miR-200a-5p, miR-200b-3p, miR-200c-3p, miR429 , and miR-25-3p could distinguish OC patients from healthy controls with AUC values of 0.649, 0.737, 0.779, 0.703 , and 0.875 , respectively (Figure $2(\mathrm{~b})$ ). The significant differences in the serum levels of miRNAs between OC patients and patients with benign ovarian diseases were reflected by miR-200a-5p, miR-200b-3p, miR-200c-3p, and miR-429 AUC values of $0.693,0.783,0.762$, and 0.692 , respectively (Figure $2(\mathrm{c})$ ). To improve the discrimination, the concentrations of these miRNAs were combined and analyzed by binary regression. One panel of these miRNAs could discriminate between OC patients and healthy controls with a sensitivity of $79.6 \%$, a specificity of $100.0 \%$, and an AUC value of 0.916 (Figure 2(b)); this panel could also differentiate OC from benign lesions with a sensitivity of $79.6 \%$ and a specificity and AUC value of 0.788 (Figure 2(c)).

3.3. Meta-Analysis of Serum miRNAs. To explore the diagnostic efficiencies of these miRNAs in OC, we performed a diagnostic meta-analysis. According to the search criteria, five articles were included in the meta-analysis $[6,8,11-13]$ and their detailed information is listed in Table 1. In total, 562 OC patients, 159 healthy women, and 65 patients with benign ovarian diseases were included in the meta-analysis.

To discriminate OC patients from healthy controls using the five miRNAs, we summarized sensitivity and specificity as 0.64 (95\% confidence interval (CI): $0.52-0.74)$ and 0.88 (95\% CI: 0.70-0.96), respectively; heterogeneity existed in both assessments $\left(P_{\text {heterogeneity }}<0.001 ; I^{2}=87.4\right.$ and $\left.90.1 \%\right)$. The SROC plot showed the summary sensitivity and specificity and the $95 \%$ confidence and prediction regions, with an AUC of 0.78 (95\% CI: 0.74-0.81, Figure 3(a)). To differentiate between OC and benign lesions (using MIR200A, MIR200B, MIR200C, and MIR429), the pooled sensitivity and specificity were 0.92 (95\% CI: $0.57-0.99)$ and 0.69 (95\% CI: $0.53-0.82)$, respectively. $I^{2}$ values for pooled sensitivity and specificity were $97.2 \%$ and $88.5 \%$, respectively, indicating 


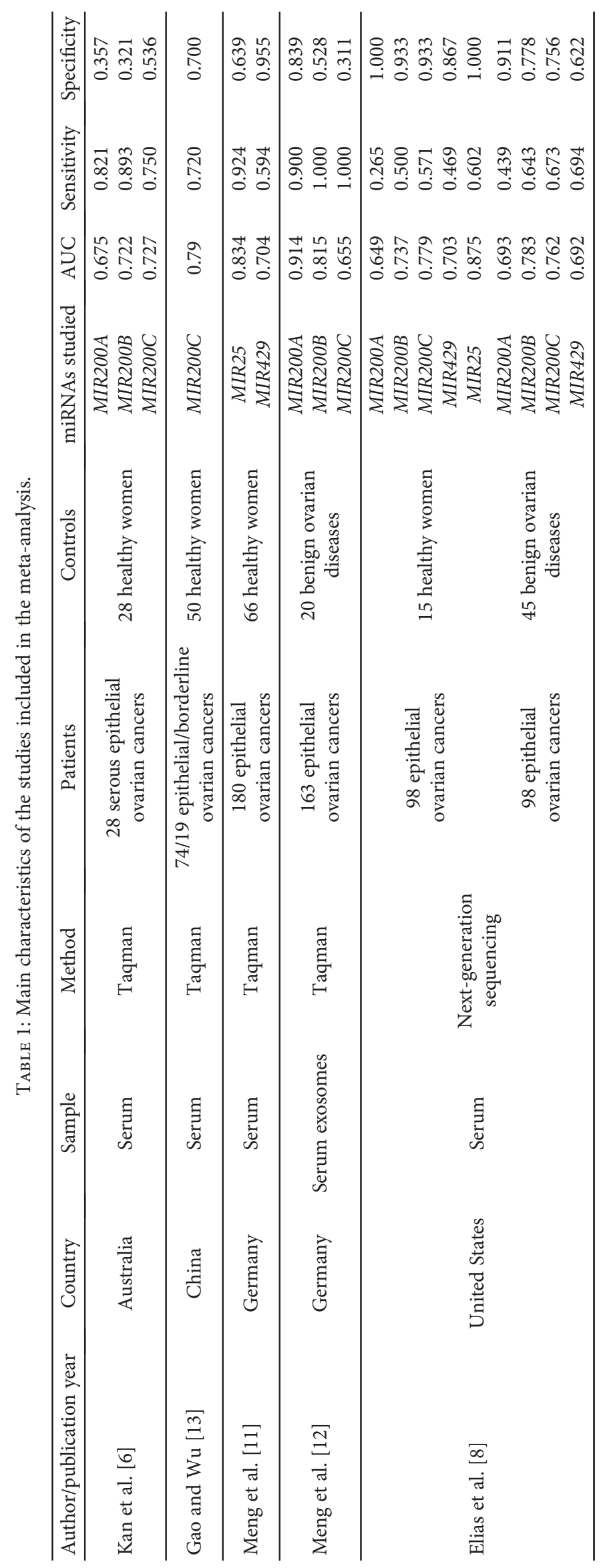



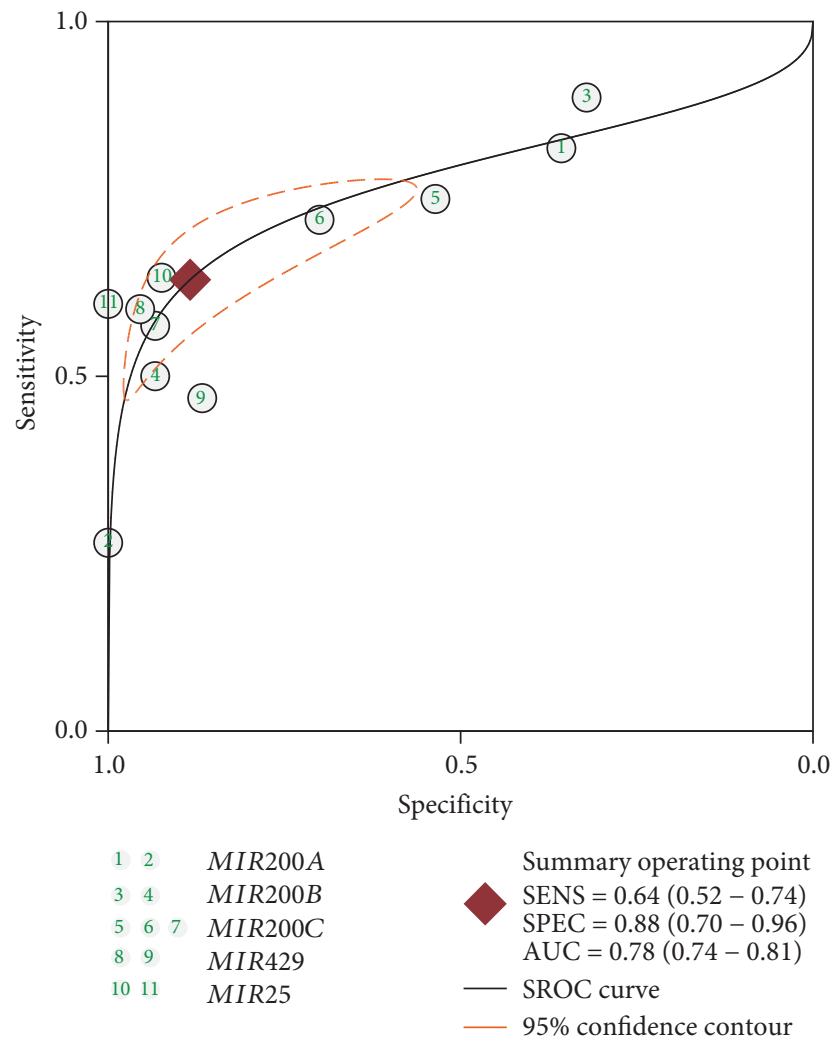

(a)

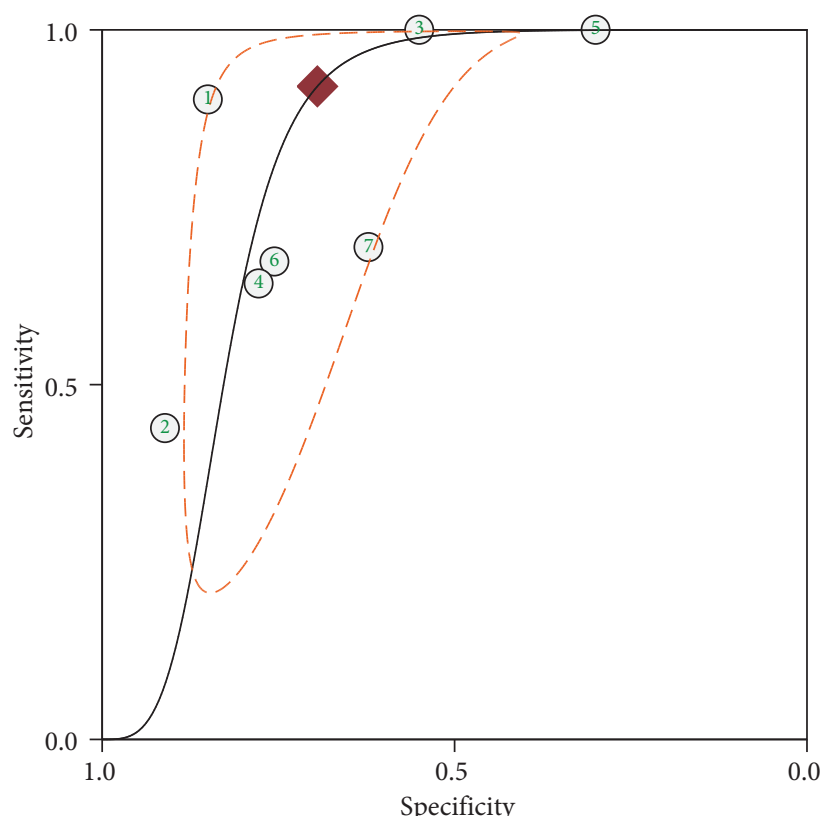
12 MIR200A
34 MIR $200 B$
56 MIR200C
Summary operating point
SENS $=0.92(0.57-0.99)$
SPEC $=0.69(0.53-0.82)$ AUC $=0.81(0.78-0.85)$
- SROC curve
_ $95 \%$ confidence contour

(b)

FIGURE 3: Summary receiver operating characteristic (SROC) curve for candidate miRNAs in the diagnosis of ovarian cancer for all studies. (a) SROC curve differentiating ovarian cancer from healthy controls. (b) SROC curve differentiating ovarian cancer from benign lesions. 
the existence of statistical heterogeneity between studies. Simultaneously, diagnostic accuracy was also assessed by SROC plotting, with an AUC value of 0.81 (95\% CI $0.78-$ 0.85 , Figure 3(b)).

For our diagnostic meta-analysis, the funnel plot of publication bias showed no asymmetry for discriminating OC patients from healthy women and the Deeks test $P$ value was 0.121 . However, substantial funnel plot asymmetry suggestive of publication bias was revealed for differentiating OC from benign lesions $(P=0.002)$, possibly because only four articles containing seven studies were analyzed.

3.4. Candidate miRNA Analysis in the Validation Set. To validate whether the five candidate miRNAs had potential as clinical biomarkers, we compared their expression in the independent set GSE106817. As shown in Figure 4(a), miR200a-5p, miR-200b-3p, miR-200c-3p, and miR-429 showing significant alterations in expression were upregulated in OC with respect to the healthy control group (all $P<0.001$ ). By contrast, miR-25-3p was significantly increased compared with healthy controls. When patients were stratified according to FIGO, miR-25-3p expression significantly decreased in stage I/II patients compared with controls (Figure 4(b)). These candidate miRNAs were also found to be aberrantly expressed in other solid tumors such as breast cancer, colorectal cancer, and gastric cancer (Figure S1). The diagnostic performance of the four miRNAs was confirmed in the validation set (AUC value: $\mathrm{miR}-200 \mathrm{a}-5 \mathrm{p}, 0.745$; $\mathrm{miR}-200 \mathrm{~b}-$ 3p, 0.690; miR-200c-3p, 0.670; and miR-429, 0.797). miR$25-3 p$ was calculated to have a sensitivity of $31.5 \%$, a specificity of $75.5 \%$, and an AUC of 0.626 (Figure 4(c)).

3.5. The Impact of Candidate miRNAs on Cellular Pathways and Biological Processes. Experimentally validated targets from miRTarBase were extracted to elucidate the biological function of these candidate miRNAs, and 167 genes were found [14]. These genes were classified into three GO categories (biological process (BP), cellular component (CC), and molecular function (MF)) using the online analysis tool DAVID. BP genes exhibited significant enrichment in the negative/positive regulation of transcription from the RNA polymerase II promoter (GO:0000122/GO:0045944, $P=$ $\left.4.04 \times 10^{-13} / 2.90 \times 10^{-11}\right)$. Among the $\mathrm{CC}$ and MF genes, the most clustered GO terms were nucleoplasm and protein binding, respectively (GO:0005654, $P=1.14 \times 10^{-10}$; GO:0005515, $\left.P=2.17 \times 10^{-15}\right)$. Regarding KEGG pathway enrichment analysis, the following three terms were identified as the most significant: miRNAs in cancer (hsa05206, $P=1.14 \times 10^{-13}$ ), prostate cancer (hsa05215, $P=4.68 \times 10^{-8}$ ), and pathways in cancer (hsa05200, $6.88 \times 10^{-8}$ ). The top 20 GO terms and enriched pathways are shown in Figure 5.

\section{Discussion}

There is emerging evidence that circulating miRNAs can be repeatedly and stably detected in the blood and serve as molecular markers in both physiological and pathological conditions for OC [15]. However, differences in measurement platforms, laboratory protocols, and small sample sizes can affect gene expression levels, so robust conclusions are rarely yielded across diverse studies. To compensate for these shortcomings, this study applied bioinformatics and metaanalysis to identify valuable circulating miRNAs in the diagnosis of OC.

Based on rigorous evaluations, we screened a total of 42 DEmiRNAs in GSE94533. Of these, MIR200A, MIR200B, MIR200C, MIR429, and MIR25 have been previously reported. In the diagnostic meta-analysis, these five circulating miRNAs demonstrated a high diagnostic accuracy and yielded a combined AUC of 0.78 with $64 \%$ pooled sensitivity and $88 \%$ pooled specificity in discriminating OC cases from healthy controls. Excluding miR-25, the remaining four miRNAs showed a combined AUC of 0.81 with $92 \%$ pooled sensitivity and $69 \%$ pooled specificity in differentiating OC cases from patients with benign disease. Similarly, we observed the same diagnostic efficiency for these four miRNAs in our independent test.

The MIR200 family contains MIR200A, MIR200B, MIR200C, and MIR429, which are generated from two distinct transcripts: MIR200A/MIR200B/MIR429 is derived from chromosome 1 and MIR200C from chromosome 12 [6]. MIR25 is located on chromosome 7 [16]. Members of the MIR200 family are reported to be highly expressed in OC, as validated in our study, suggesting their importance in the diagnosis of OC. The MIR200 family is thought to play an essential role in tumor metastasis by promoting epithelialmesenchymal transition (EMT). MIR200 family members help maintain E-cadherin expression in OC by downregulating ZEB1 and ZEB2, which are key transcription factors in EMT mediation that act as known repressors of E-cadherin transcription [17].

Decreased serum levels of MIR25 were detected in GSE94533 in the present study, which is consistent with recent findings by Langhe et al. [7] and Meng et al. [11]. However, this phenomenon was only observed in earlystage OC samples in the GSE106817 set, which could reflect the different methods used for miRNA extraction and detection. Nevertheless, data on the effect of this miRNA in OC remain contradictory. miR-25 was previously reported to be significantly upregulated in OC compared with the healthy ovarian tissue $[16,18]$. Additionally, MIR25 was reported to interact with large numbers of protein-coding genes (ITGA5, FBN1, and CDH1) and noncoding genes (lncRNA PTAF) whose expression changes promote ovarian carcinogenesis $[18,19]$. Interestingly, Benson et al. detected a significantly decreased circulating MIR25 concentration (-1.82-fold) in OC patients who had undergone carboplatin chemotherapy compared to subjects before treatment, which indicated that its change was associated with clinical response [20].

To determine the potential target genes of these miRNAs, we conducted functional and signaling pathway analysis. The top 20 enriched GO terms and signaling pathways were shown to be involved in the development and prognosis of cancer, such as focal adhesion and the phosphoinositide 3kinase/Akt signaling pathway. Many miRNAs have been found to influence this pathway which is considered to play an instrumental role in proliferation, migration, invasion, and chemotherapy resistance [21]. Focal adhesion is a 


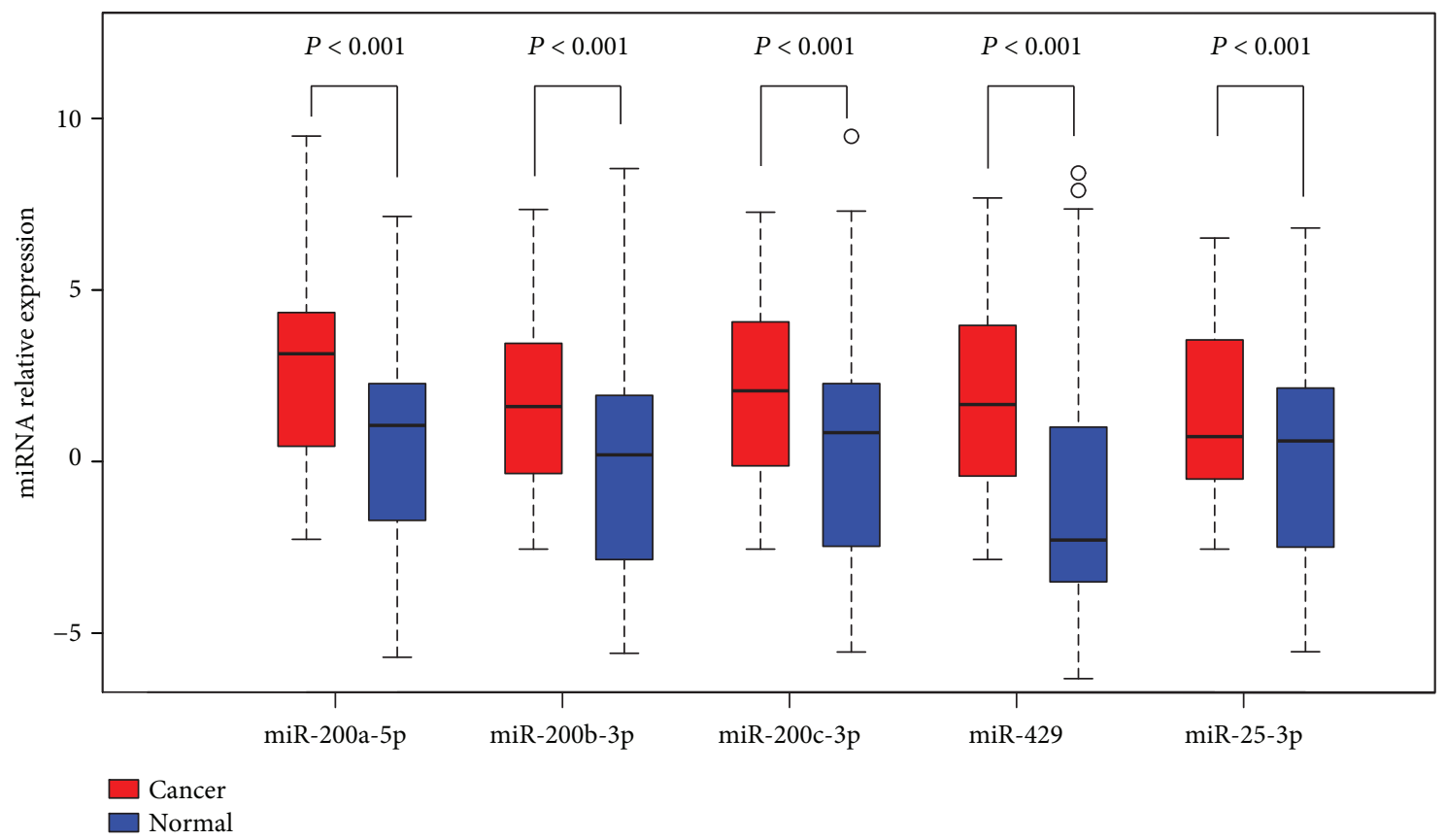

(a)
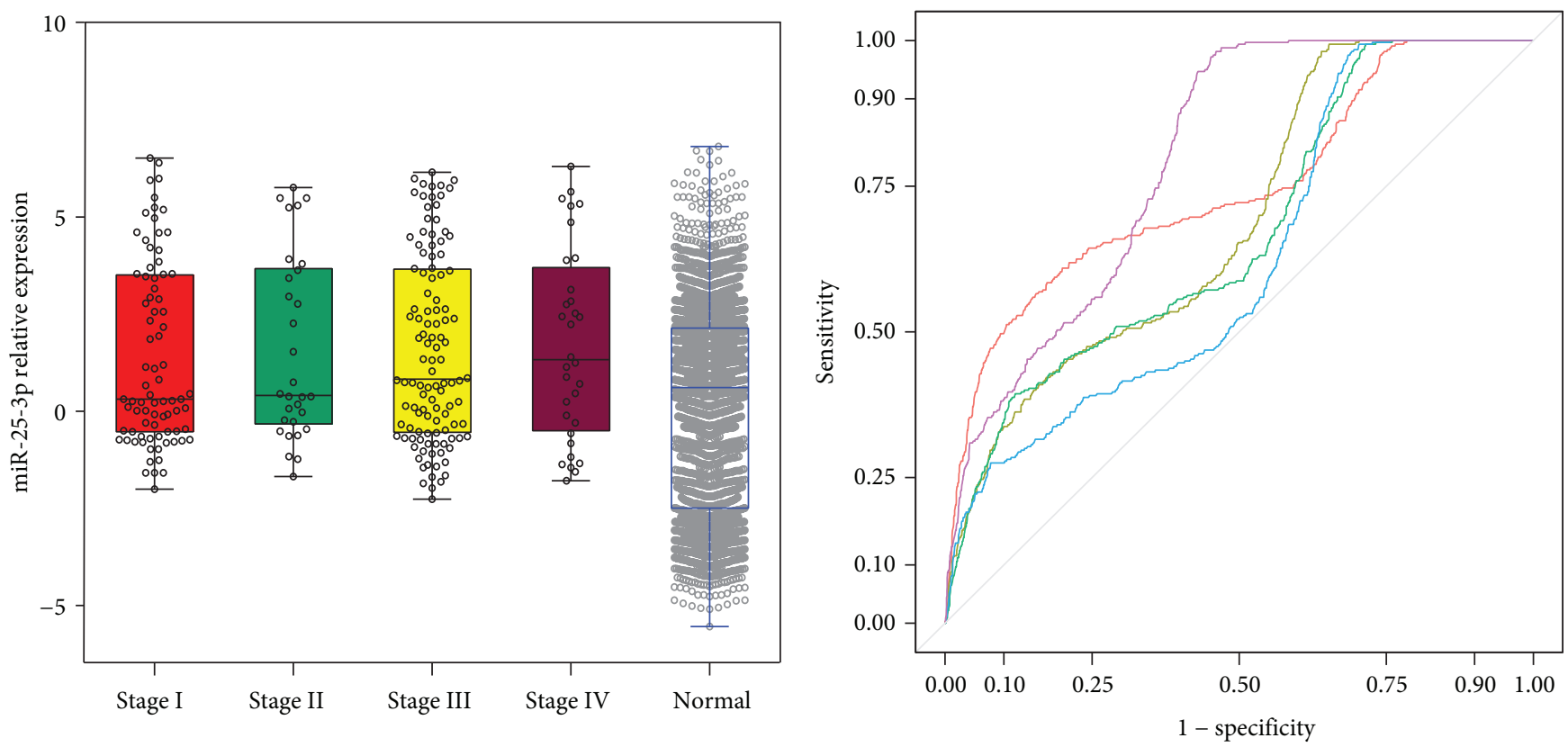

AUC

$\begin{array}{ll}\text { miR-200a-5p } & 0.745 \\ \text { miR-200b-3p } & 0.690 \\ \text { miR-200c-3p } & 0.670 \\ \text { miR-429 } & 0.797 \\ \text { miR-25-3p } & 0.626\end{array}$

(b)

(c)

FIGURE 4: Quantification of candidate miRNAs in the validation set. (a) Box plot comparing miRNA concentrations in the serum of healthy women and patients with ovarian cancer. (b) The relative expression of miR-25-3p in the serum of healthy women and ovarian cancer patients stratified by tumor grade. (c) Receiver operating characteristic curve for candidate miRNAs showing its potential to discriminate ovarian cancer patients from healthy women. 


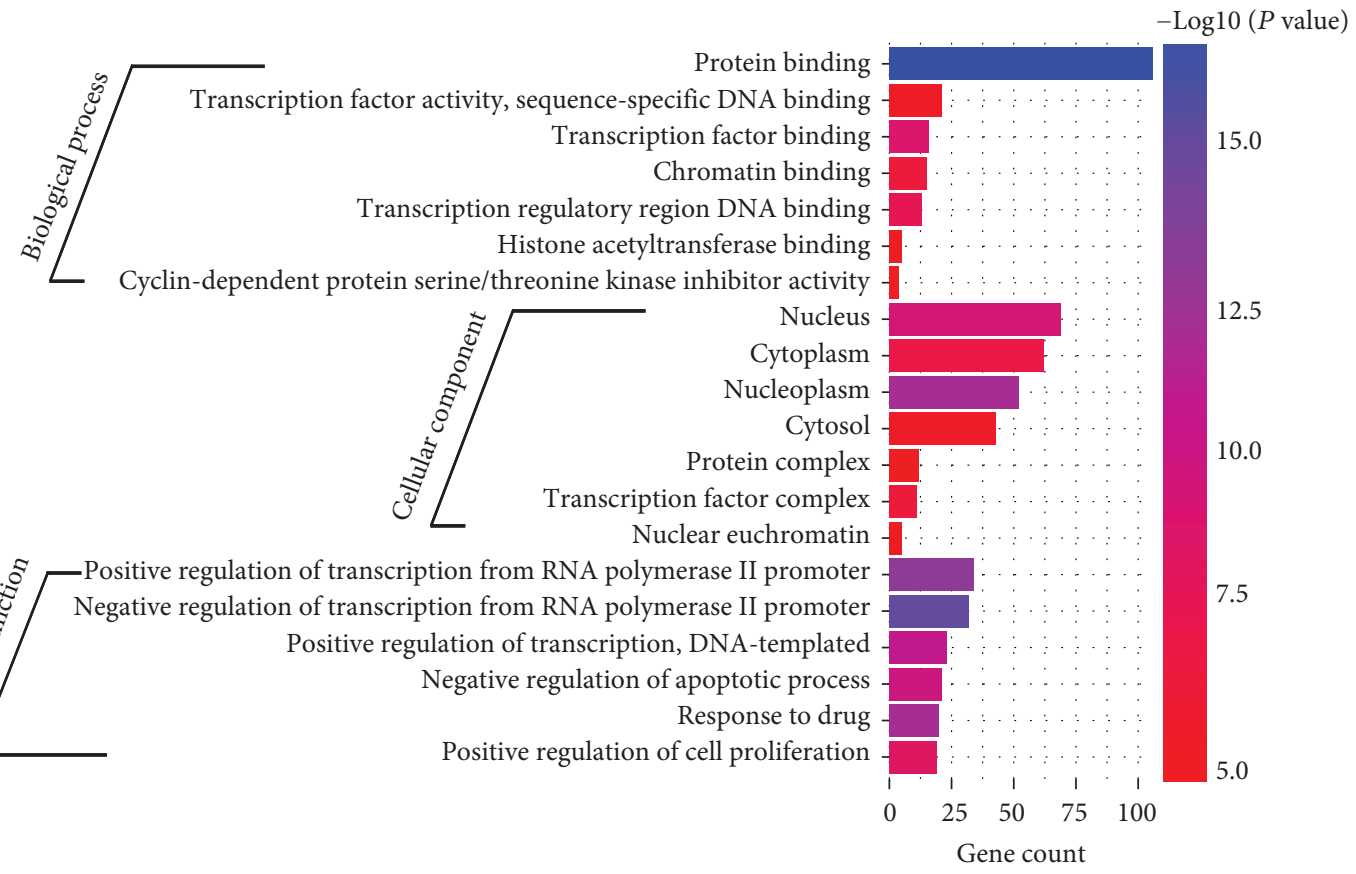

(a)

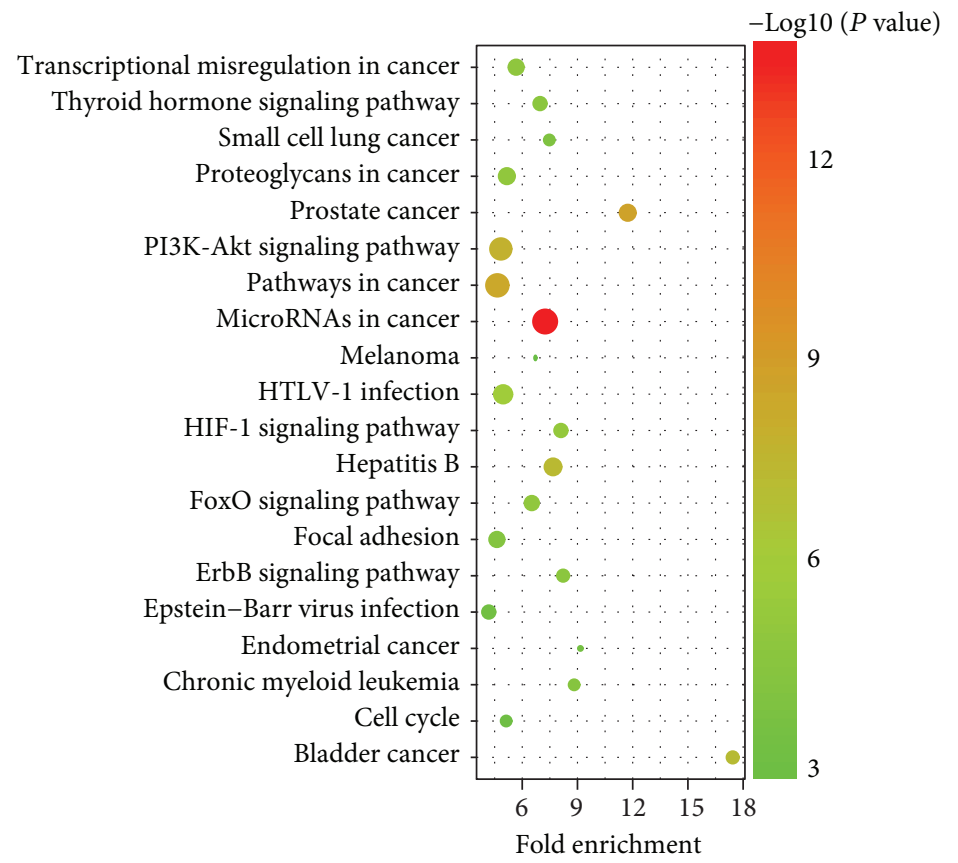

Gene number

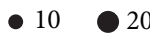

$15 \bigcirc 25$

(b)

FIGURE 5: Functional annotation of the predicted targets of candidate miRNAs. (a) The top 20 GO terms derive from the "biological process," "cellular component," and "molecular function" categories by GO analysis. (b) The top 20 saturated pathways are generated from KEGG pathway analysis.

common mechanism associated with tumor cell invasion and metastasis, including that of OC [22]. Therefore, taken together with previous findings, the varied functions of these miRNAs imply that they have the capacity to interact with several targets and effect mechanistic changes.
The expression of miRNAs in serum or plasma is fairly stable and can be detected using common laboratory methods. This may be because circulating miRNAs are protected by binding proteins or are chemically modified, such as by methylation, making them resistant to ribonuclease 
activity $[23,24]$. Some researchers have proposed that circulating miRNAs are derived from the secretion or leakage of microvesicles, exosomes, or apoptotic bodies from healthy and tumor tissues [25]. This provides further support for OC-associated circulating miRNA expression profiles as indicators of biological function.

Our study has a number of strengths. The first is that we performed in silico analysis to investigate the diagnosis value and biochemical properties of circulating miRNAs in OC. Second, we included controls of patients with benign disease and healthy individuals. Third, the MIR200 family with its high combined AUC and specificity was demonstrated to be a better molecular marker for OC prediction than MIR25, especially in the validation set. Fourth, we used a combination of bioinformatics and meta-analysis to identify OC biomarkers. However, we also recognize our study limitations, including the small sample size, selection bias, and heterogeneity across studies.

\section{Conclusions}

Our comprehensive analysis identified circulating MIR200 family members as promising noninvasive screening tools for the early detection of OC. Further large-scale prospective studies are warranted to confirm the clinical relevance of these miRNAs.

\section{Data Availability}

The raw data of next-generation sequencing in this study are available in the NCBI database (https://www.ncbi.nlm .nih.gov/geoprofiles/) under accession number GSE94533. The microarray data that support this study are available through the NCBI database under accession GSE106817. The data used to support the findings of this study are included within the article.

\section{Conflicts of Interest}

The authors declare that they have no conflict of interest.

\section{Acknowledgments}

This work was supported by the grants from the Natural Science Foundation of Education Committee of Nanjing Medical University (YB2017075).

\section{Supplementary Materials}

Figure S1: The relative expression levels of 5 identified miRNAs in serum samples from patients with different types of tumors and healthy women: (a) miR-200a-5p, (b) miR200b-3p, (c) miR-200c-3p, (d) miR-429, and (e) miR-25-3p. (Supplementary Materials)

\section{References}

[1] R. L. Siegel, K. D. Miller, and A. Jemal, "Cancer statistics, 2017,” CA: A Cancer Journal for Clinicians, vol. 67, no. 1, pp. 7-30, 2017.
[2] U. Menon, M. Griffin, and A. Gentry-Maharaj, "Ovarian cancer screening-current status, future directions," Gynecologic Oncology, vol. 132, no. 2, pp. 490-495, 2014.

[3] O. Blyuss, M. Burnell, A. Ryan et al., "Comparison of longitudinal Ca125 algorithms as a first-line screen for ovarian cancer in the general population," Clinical Cancer Research, vol. 24, no. 19, pp. 4726-4733, 2018.

[4] X. J. Lin, Y. Chong, Z. W. Guo et al., "A serum microRNA classifier for early detection of hepatocellular carcinoma: a multicentre, retrospective, longitudinal biomarker identification study with a nested case-control study," The Lancet Oncology, vol. 16, no. 7, pp. 804-815, 2015.

[5] D. D. Taylor and C. Gercel-Taylor, "MicroRNA signatures of tumor-derived exosomes as diagnostic biomarkers of ovarian cancer," Gynecologic Oncology, vol. 110, no. 1, pp. 13-21, 2008.

[6] C. W. Kan, M. A. Hahn, G. B. Gard et al., "Elevated levels of circulating microRNA-200 family members correlate with serous epithelial ovarian cancer," BMC Cancer, vol. 12, no. 1, p. $627,2012$.

[7] R. Langhe, L. Norris, F. A. Saadeh et al., "A novel serum microRNA panel to discriminate benign from malignant ovarian disease," Cancer Letters, vol. 356, no. 2, pp. 628-636, 2015.

[8] K. M. Elias, W. Fendler, K. Stawiski et al., "Diagnostic potential for a serum miRNA neural network for detection of ovarian cancer," eLife, vol. 6, 2017.

[9] A. Yokoi, J. Matsuzaki, Y. Yamamoto et al., "Integrated extracellular microRNA profiling for ovarian cancer screening," Nature Communications, vol. 9, no. 1, p. 4319, 2018.

[10] C. M. Rutter and C. A. Gatsonis, "A hierarchical regression approach to meta-analysis of diagnostic test accuracy evaluations," Statistics in Medicine, vol. 20, no. 19, pp. 2865-2884, 2001.

[11] X. Meng, S. A. Joosse, V. Müller et al., "Diagnostic and prognostic potential of serum Mir-7, Mir-16, Mir-25, Mir-93, Mir-182, Mir-376a and Mir-429 in ovarian cancer patients," British Journal of Cancer, vol. 113, no. 9, pp. 1358-1366, 2015.

[12] X. Meng, V. Müller, K. Milde-Langosch, F. Trillsch, K. Pantel, and H. Schwarzenbach, "Diagnostic and prognostic relevance of circulating exosomal Mir-373, Mir-200a, Mir-200b and Mir-200c in patients with epithelial ovarian cancer," Oncotarget, vol. 7, no. 13, pp. 16923-16935, 2016.

[13] Y. C. Gao and J. Wu, "MicroRNA-200c and microRNA-141 as potential diagnostic and prognostic biomarkers for ovarian cancer," Tumour Biology, vol. 36, no. 6, pp. 4843-4850, 2015.

[14] C. H. Chou, S. Shrestha, C. D. Yang et al., "miRTarBase update 2018: a resource for experimentally validated microRNAtarget interactions," Nucleic Acids Research, vol. 46, no. D1, pp. D296-D302, 2018.

[15] A. R. Halvorsen, G. Kristensen, A. Embleton et al., "Evaluation of prognostic and predictive significance of circulating microRNAs in ovarian cancer patients," Disease Markers, vol. 2017, Article ID 3098542, 9 pages, 2017.

[16] X. Wang, X. Meng, H. Li, W. Liu, S. Shen, and Z. Gao, "MicroRNA-25 expression level is an independent prognostic factor in epithelial ovarian cancer," Clinical \& Translational Oncology, vol. 16, no. 11, pp. 954-958, 2014.

[17] X. Hu, D. M. Macdonald, P. C. Huettner et al., "A Mir-200 microRNA cluster as prognostic marker in advanced ovarian cancer," Gynecologic Oncology, vol. 114, no. 3, pp. 457-464, 2009. 
[18] H. Liang, X. Zhao, C. Wang et al., "Systematic analyses reveal long non-coding RNA (PTAF)-mediated promotion of EMT and invasion-metastasis in serous ovarian cancer," Molecular Cancer, vol. 17, no. 1, p. 96, 2018.

[19] D. Yang, Y. Sun, L. Hu et al., "Integrated analyses identify a master microRNA regulatory network for the mesenchymal subtype in serous ovarian cancer," Cancer Cell, vol. 23, no. 2, pp. 186-199, 2013.

[20] E. A. Benson, T. C. Skaar, Y. Liu, K. P. Nephew, and D. Matei, "Carboplatin with decitabine therapy, in recurrent platinum resistant ovarian cancer, alters circulating miRNAs concentrations: a pilot study," PLoS One, vol. 10, no. 10, article e0141279, 2015

[21] M. L. Gasparri, Z. M. Besharat, A. A. Farooqi et al., "MiRNAs and their interplay with $\mathrm{PI} 3 \mathrm{~K} / \mathrm{AKT} / \mathrm{mTOR}$ pathway in ovarian cancer cells: a potential role in platinum resistance," Journal of Cancer Research and Clinical Oncology, vol. 144, no. 12, pp. 2313-2318, 2018.

[22] J. Halder, Y. G. Lin, W. M. Merritt et al., "Therapeutic efficacy of a novel focal adhesion kinase inhibitor Tae226 in ovarian carcinoma," Cancer Research, vol. 67, no. 22, pp. 1097610983, 2007.

[23] T. Muraoka, J. Soh, S. Toyooka et al., “The degree of microRNA-34b/C methylation in serum-circulating DNA is associated with malignant pleural mesothelioma," Lung Cancer, vol. 82, no. 3, pp. 485-490, 2013.

[24] C. Zhang, C. Wang, X. Chen et al., "Expression profile of microRNAs in serum: a fingerprint for esophageal squamous cell carcinoma," Clinical Chemistry, vol. 56, no. 12, pp. 18711879, 2010.

[25] K. Nakamura, K. Sawada, A. Yoshimura, Y. Kinose, E. Nakatsuka, and T. Kimura, "Clinical relevance of circulating cell-free microRNAs in ovarian cancer," Molecular Cancer, vol. 15, no. 1, p. 48, 2016. 


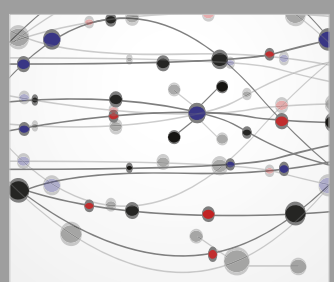

The Scientific World Journal
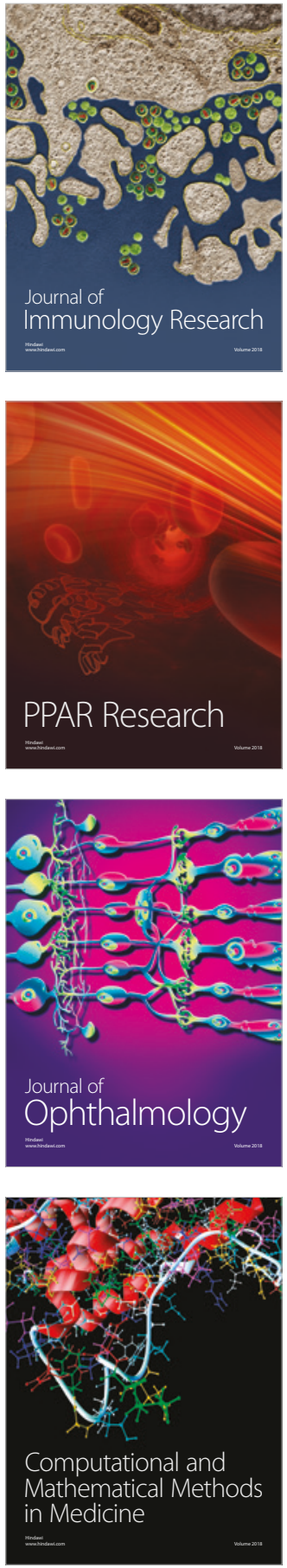

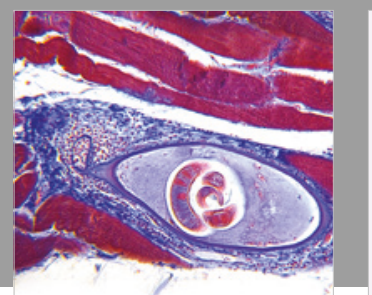

Gastroenterology Research and Practice

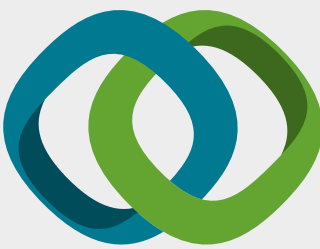

\section{Hindawi}

Submit your manuscripts at

www.hindawi.com
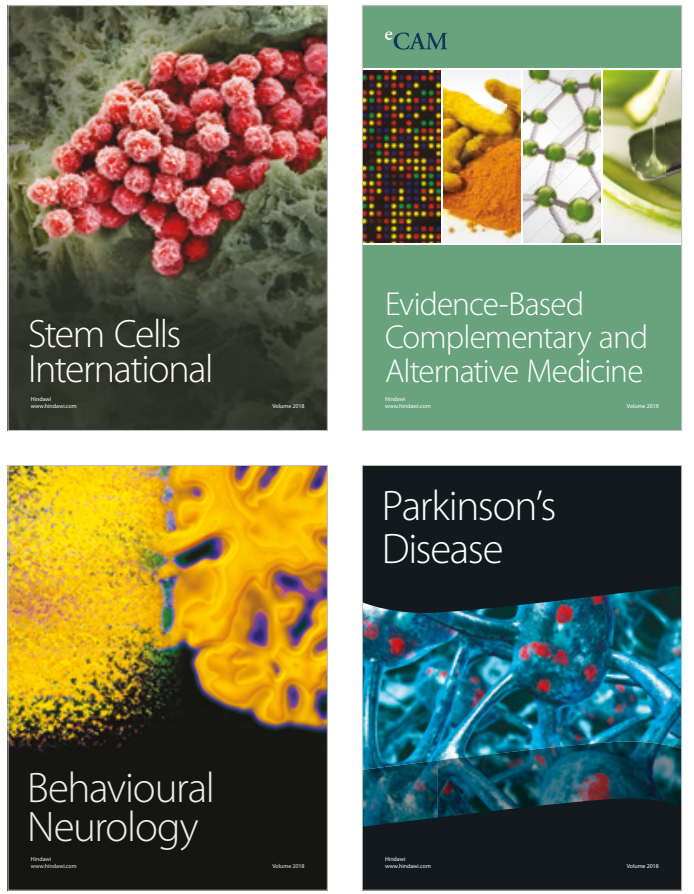

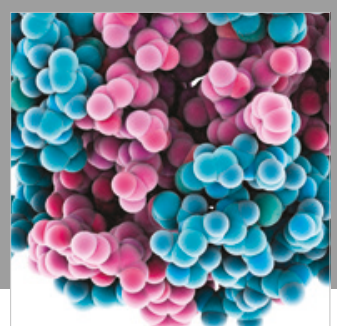

ournal of

Diabetes Research

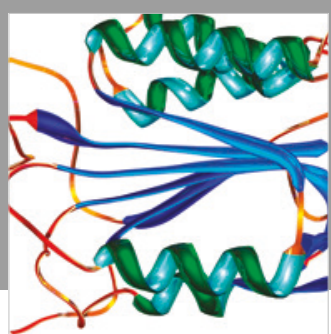

Disease Markers
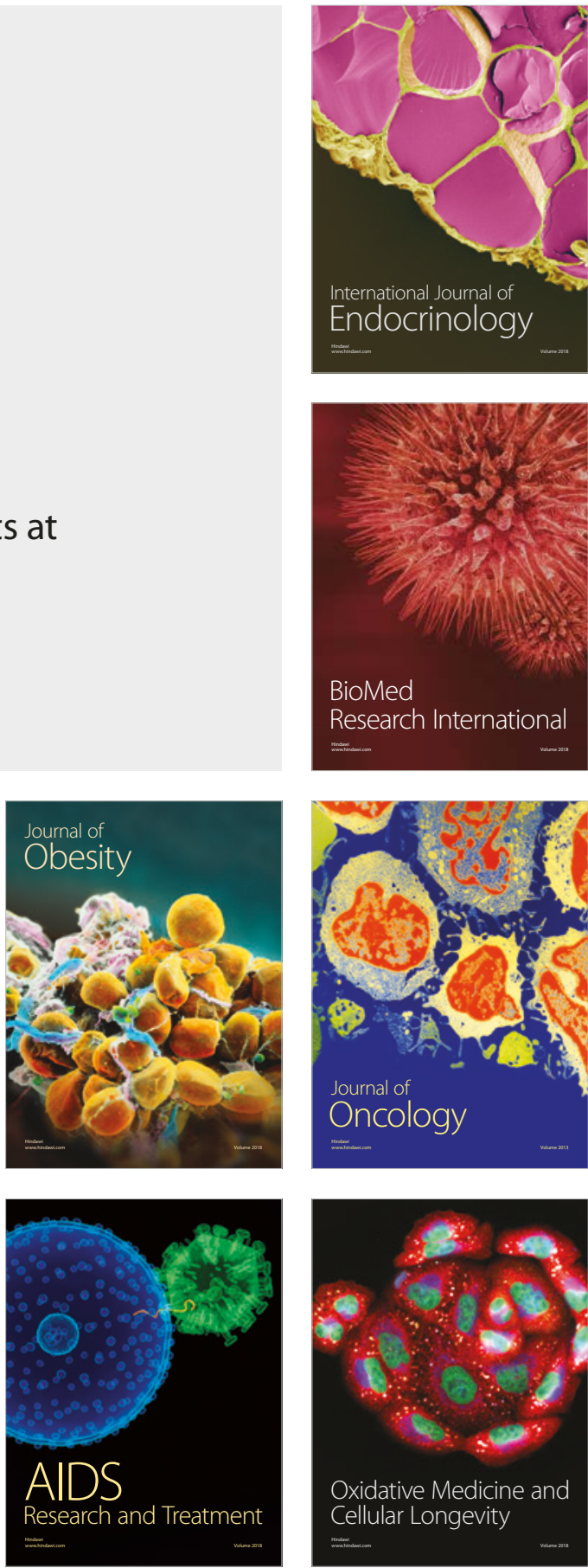